\title{
The RICH detector of the NA62 experiment
}

\author{
Viacheslav Duk* \\ INFN Perugia \\ Via A. Pascoli, Perugia, 06123, Italy \\ Viacheslav.Duk@cern.ch
}

Published 1 September 2016

\begin{abstract}
The NA62 experiment at CERN is aimed at measuring the ultra-rare decay $\mathrm{K}^{+} \rightarrow \pi^{+} v v$ with $10 \%$ accuracy. One of the detectors that is crucial for the rejection of background events is the RICH detector: a gas based detector aimed at $\pi / \mu$ separation in the $15-35 \mathrm{GeV} / \mathrm{c}$ momentum range with an inefficiency of less than $1 \%$. The RICH must also provide a very precise time measurement (with the time resolution $\sim 100 \mathrm{ps}$ ) to correctly associate the $\pi^{+}$with the parent $\mathrm{K}^{+}$particle measured by an upstream detector. This paper contains the detailed description of the RICH detector, its readout, and the results of the commissioning run at CERN in 2014.
\end{abstract}

Keywords: kaon decays; RICH detectors; NA62 experiment.

\section{NA62 Experiment at CERN}

The major goal of the NA62 experiment at CERN SPS ${ }^{1,2}$ is to measure the branching ratio (BR) of the ultra-rare decay $K^{+} \rightarrow \pi^{+} v \bar{v}$ with $\sim 10 \%$ accuracy. The detector layout is shown in Fig. 1. Since the branching fraction of this decay is $\mathrm{O}\left(10^{-10}\right)$, the detector must be able to suppress background events with BR up to 10 orders of magnitude higher than the signal, such as $\mathrm{K}^{+} \rightarrow \mu^{+} v_{\mu}(\mathrm{BR} \sim 64 \%)$ and $\mathrm{K}^{+} \rightarrow \pi^{+} \pi^{0} \quad(\mathrm{BR} \sim 21 \%)$. One of the crucial detectors to suppress the $\mathrm{K}^{+} \rightarrow \mu^{+} v_{\mu}$ background is the RICH (Ring Imaging Cherenkov detector): a gas based detector aimed at $\pi / \mu$ separation in the $15-35 \mathrm{GeV} / \mathrm{c}$ momentum range with an inefficiency of less than $1 \%$.

\footnotetext{
${ }^{*}$ For the RICH working group of the NA62 experiment: INFN Perugia, INFN Florence, CERN.

$\dagger$ Istituto Nazionale di Fisica Nucleare, Sezione di Perugia. Via A. Pascoli, Perugia, 06123, Italy.
}

This is an Open Access article published by World Scientific Publishing Company. It is distributed under the terms of the Creative Commons Attribution 3.0 (CC-BY) License. Further distribution of this work is permitted, provided the original work is properly cited. 


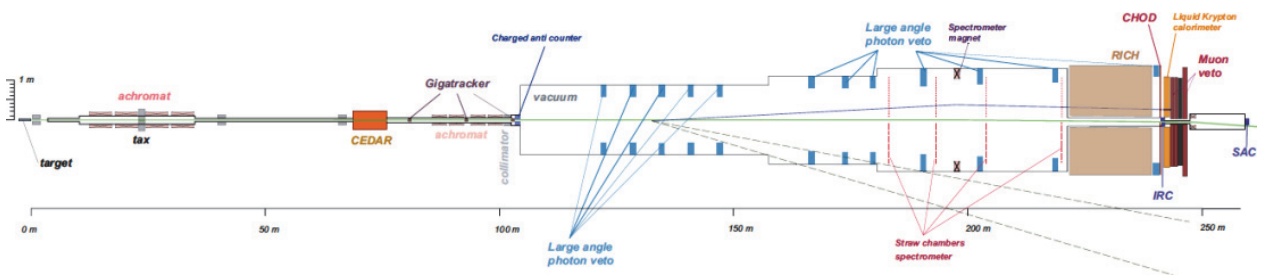

Fig. 1. Layout of the NA62 experimental setup.

\section{The RICH Detector}

The RICH detector has the following requirements:

- Provide a muon suppression in the pion sample at the $\sim 10^{-2}$ level in the momentum range $15 \mathrm{GeV} / \mathrm{c}<\mathrm{p}<35 \mathrm{GeV} / \mathrm{c}$.

- Measure the pion crossing time with a resolution of $\sim 100$ ps.

- $\quad$ Produce an L0 trigger pulse for a secondary charged track.

These requirements drive the choice of the main detector characteristics discussed in more detail below.

\subsection{Vessel}

The RICH detector consists of a $17.5 \mathrm{~m}$ long vacuum proof cylindrical vessel shown in Fig. 2. The outer diameter varies from $3.4 \mathrm{~m}$ to $4 \mathrm{~m}$. The vessel is filled with neon at atmospheric pressure and is at room temperature. It was installed at CERN in February 2014; the full vacuum test was done in March 2014.

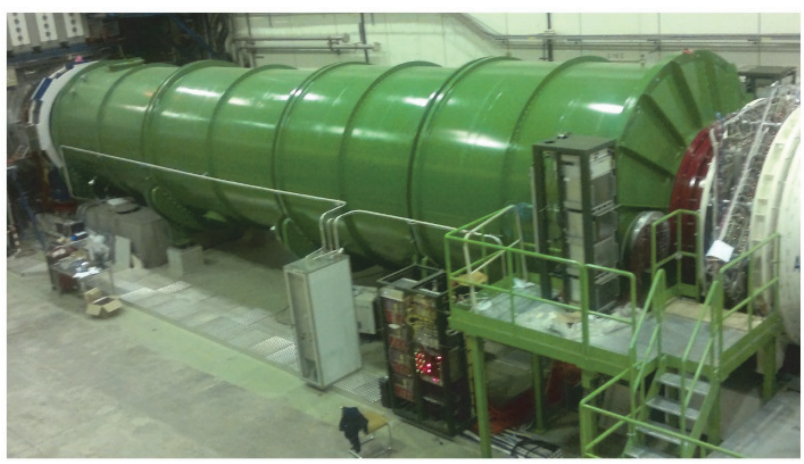

Fig. 2. RICH vessel. 


\subsection{Mirrors}

Mirrors with $17 \mathrm{~m}$ focal length reflecting the Cherenkov light are placed at the downstream end of the vessel. A mirror mosaic, shown in Fig. 3, covers the area of $\sim 3 \mathrm{~m}$ in diameter. The mosaic is composed of 18 spherical mirrors of hexagonal shape ( 350 mm side) and 2 of semi-hexagonal shape around the beam pipe.
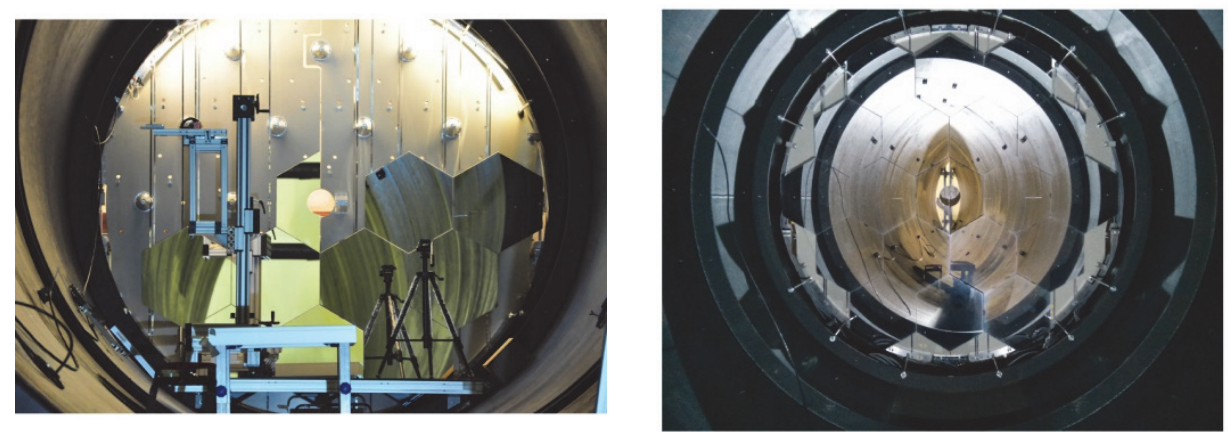

Fig. 3. Left: mirrors installed in the lower part of the mosaic inside the RICH; the loading tool is visible on the picture. Right: the completed mirror mosaic.

The mirrors are made of $2.5 \mathrm{~cm}$ thick glass and coated with aluminum. A thin dielectric film is added to improve the reflectivity, which is more than $90 \%$ in the range $\lambda=195-650 \mathrm{~nm}$. The mirror support system is a $5 \mathrm{~cm}$ thick honeycomb structure. The mirrors are supported by a dowel and oriented in space by means of three aluminum ribbons (two ribbons to tune the mirror orientation and one stabilizing ribbon to prevent its rotation). The ribbons are moved by means of piezo motors that are operated remotely.

\subsection{Light collection}

The reflected light is collected by 2000 photomultipliers (PMs) (Hamamatsu R7400 U03) assembled in a compact hexagonal packing into two aluminum discs at the entrance window as seen in Fig. 4. Winston cones carved into the discs and covered with aluminized Mylar are used to bring the light to the PM active area. The Mylar increases the light collection by a factor of $\sim 20 \%$. Quartz windows are used to separate the PMs from the neon. 

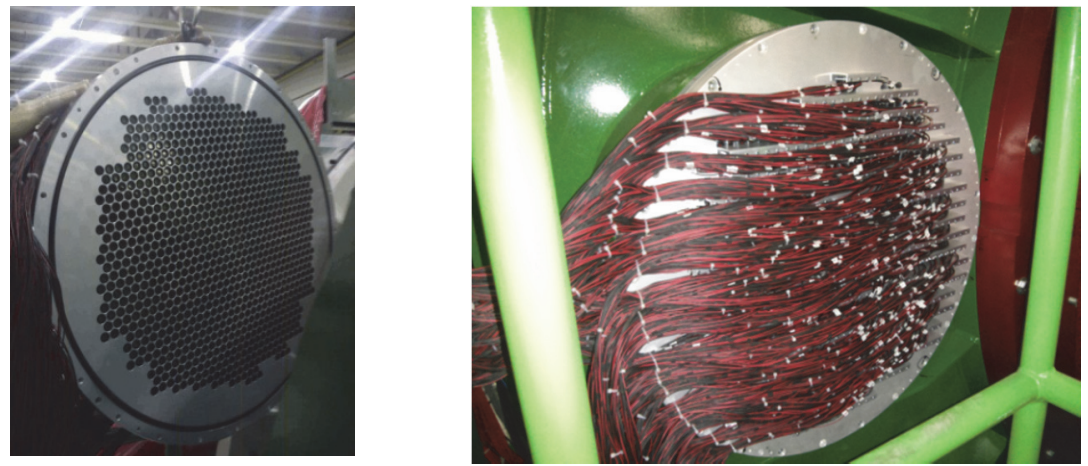

Fig. 4. One of two aluminum discs for PMs: before (left) and after (right) the PM installation.

\subsection{Front-end electronics}

The signals from the PMs are sent to custom-made current amplifiers with differential output and then to NINO chips used as discriminators operating in time-over-threshold mode. Both amplifiers and NINO chips are integrated in the front-end (FE) electronics boards that also include an ELMB (embedded local monitor board, developed by the ATLAS experiment ${ }^{3}$ ) for the remote control and monitoring of the FE parameters, and a multiplicity output. Each FE board contains 32 channels; in total there are 64 boards.

\subsection{Readout system}

The data acquisition system is based on TEL62 boards ${ }^{4}$ of the same type used by other NA62 detectors. Each board can house up to four 128-channel daughter boards called TDCBs. Four TEL62 boards are used for the signals from 1952 PMs and one for a multiplicity output from the front-end readout to produce an L0 trigger signal.

\section{RICH Performance in the Commissioning Run}

Most detectors of the NA62 setup were commissioned in the two-month run in OctoberDecember, 2014 . The beam intensity during the run was $\sim 5 \%$ of the nominal (750 MHz), and the track information was not available during the run.

To study the RICH performance, a sample of positive pions was selected from $\mathrm{K}^{+} \rightarrow$ $\pi^{+} \pi^{0}$ decays: the nominal beam momentum $(75 \mathrm{GeV} / \mathrm{c})$ was used for $\mathrm{K}^{+}$, the $\pi^{0}$ momentum was reconstructed using the information from the electromagnetic calorimeter $\operatorname{LKr}$ (see Ref. 2 for details), and the $\pi^{+}$momentum was calculated from the kinematics of the decay. Figure 5 shows the PM flange illuminations by the Cherenkov light from $\pi^{+}$; the flanges are named "Jura" and "Saleve". 

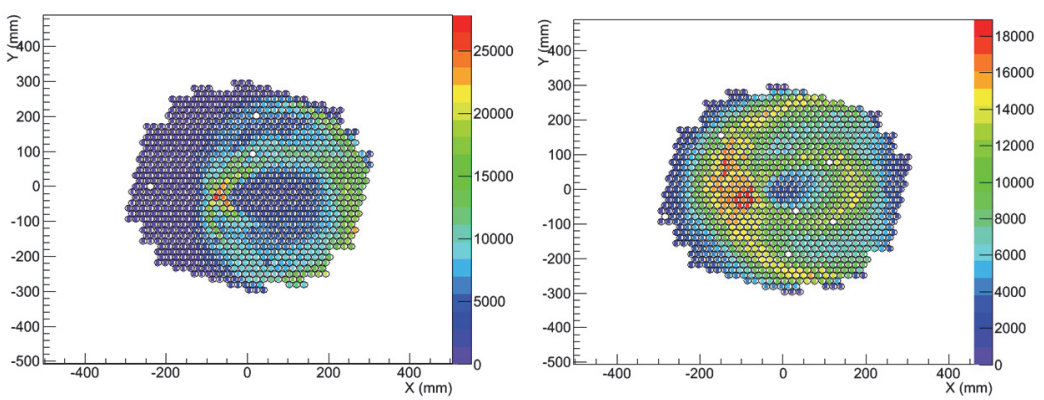

Fig. 5. PM flange illumination ("Jura" on the left, "Saleve" on the right).

Figure 6 illustrates the number of hits per Cherenkov ring as a function of the pion momentum and the distribution of the Cherenkov ring radius vs particle momentum.
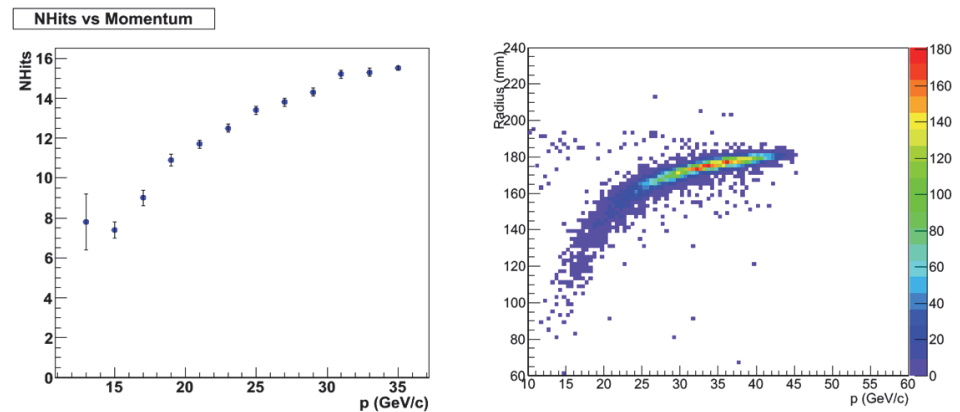

Fig. 6. Left: multiplicity vs particle momentum. Right: ring radius vs particle momentum.

The independent measurement of the RICH time resolution was done in the following way:

- Hits were split into two groups.

- Average time was calculated for each group.

- The difference of average times was fitted by a Gaussian.

- The RICH time resolution is $\sim 0.5^{*} \sigma$, where $\sigma$ is a fit parameter.

The fit of the average time difference plot is shown in Fig. 7. The RICH event time resolution is about $70 \mathrm{ps}$. The preliminary results of the commissioning run indicate that the RICH performance satisfies the NA62 experiment requirements. 


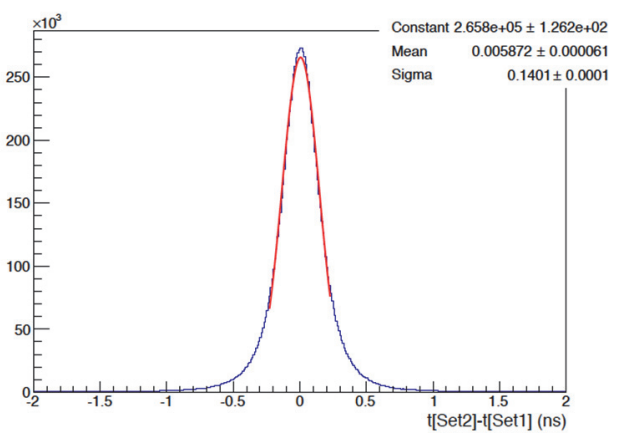

Fig. 7. Difference of average times of two groups of hits in the same Cherenkov ring. A Gaussian fit is superimposed (red curve).

\section{Conclusions}

The RICH detector of the NA62 experiment plays a key role in the particle ID, time measurement of a secondary charged particle, and an L0 trigger formation. The detector was successfully commissioned during the 2014 run. The preliminary data analysis shows a reasonable performance in agreement with the experiment requirements.

\section{References}

1. G. Anelli, et al., CERN-SPSC-2005-013, CERN-SPSC-P-326 (2005).

2. F. Hahn, et al., NA62 Technical Design Document, NA62-10-07 (2010).

3. B. Hallgren, et al., The embedded local monitor board (ELMB) in the LHC front-end I/O control system, in Proc.7th Workshop Electronics for LHC Experiments (2001), p. 325, http://cdsweb.cern.ch/record/530675.

4. B. Angelucci, et al., JINST 9, C01055 (2014). 